\title{
Additions to the checklist of lichenicolous fungi of Mongolia
}

\author{
Mikhail P. Zhurbenko', Ochirbat Enkhtuya ${ }^{2} \&$ Samiya Javkhlan ${ }^{2}$ \\ ${ }^{1}$ Laboratory of the Systematics and Geography of Fungi, Komarov Botanical Institute, Russian Academy of Sciences, \\ Professora Popova 2, 197376 St. Petersburg, Russia. E-mail: zhurb58@gmail.com \\ ${ }^{2}$ Laboratory of Flora and Plant Systematics, Botanic Garden and Research Institute of Mongolian Academy of Sciences, \\ Enkhtaivan avenue 54a, Bayanzurkh district, Ulaanbaatar-13330, Mongolia. \\ E-mails: tuyabot@yahoo.com, jawhaabot@gmail.com
}

\begin{abstract}
Seventy-four species of lichenicolous fungi, including two species of lichenicolous lichens, are reported from Mongolia. Arthonia xanthoparmeliarum, Katherinomyces cetrariae s. lat. and Lichenochora arctica are newly reported for Asia. Arthonia epiphyscia, A. excentrica, A. molendoi, Carbonea vitellinaria, Cercidospora xanthoriae, Didymocyrtis rhizoplacae, Endococcus propinquus, Intralichen christiansenii, Lichenochora caloplacae, L. rinodinae, Lichenodiplis lecanorae, Lichenostigma dimelaenae, L. triseptatum, Niesslia peltigericola, Polycoccum trypethelioides, Rhymbocarpus neglectus, Sphaerellothecium phaeorrhizae, S. propinquellum, Stigmidium xanthoparmeliarum, Taeniolella pertusariicola and Tetramelas phaeophysciae are reported new to Mongolia. The occurrence of Stigmidium psorae in Mongolia is confirmed. Flavocetraria and Rhizoplaca are reported as new host genera for Katherinomyces cetrariae s. lat.
\end{abstract}

Keywords: lichen parasites, biodiversity, biogeography, Central Asia

\section{INTRODUCTION}

Recently, the first synopsis of lichenicolous fungi of Mongolia was published, which included data on 114 species, mainly collected in the Khubsugul Aimak (Zhurbenko et al., 2019). The present contribution presents the results of further studies of the lichenicolous mycobiota of Mongolia performed by the authors in 2019, mainly in the Mongolian Altai. Here, data on new finds of 74 species of lichenicolous fungi are presented, three of which are new to Asia and 21 are new to Mongolia, which increases the number of lichenicolous fungi species documented in Mongolia to 138.

\section{MATERIAL AND METHODS}

The study is based on 153 specimens of lichenicolous fungi collected by the authors from 8 to 27 July 2019 in 15 localities in Mongolia. Microscopical examination was carried out using a Stemi 2000-CS dissecting microscope and a Zeiss Axio Imager A1 compound microscope with interference contrast, fitted with an AxioCam MRc5 digital camera. Hand-cut, razor-blade sections of fruit bodies were studied in water, $10 \% \mathrm{KOH}$, Lugol's iodine, directly or after a $\mathrm{KOH}$ pre-treatment, or brilliant cresyl blue. For the identification of host lichens, commercial bleach and a solution of paraphenylenediamine in ethanol were used. The length and breadth of asci, ascospores and conidia are given (where $\mathrm{n}>$ 10) as (min-) $\{\mathrm{X}-\mathrm{SD}\}-\{\mathrm{X}+\mathrm{SD}\}(-\max )$, where "min" and "max" are the extreme observed values, $X$ the arithmetic mean and SD the corresponding standard deviation, followed by the number of measurements (n). The length/breadth ratio is indicated as L/B and given in the same way. Measurements were taken from water mounts. Geographical names mainly follow Enhbayaryn (2004). Voucher specimens are housed in the mycological herbarium of the V. L. Komarov Botanical Institute in St. Petersburg, Russia (LE).

\section{Collecting localities}

Visited localities in Mongolia are arranged in chronological order and referenced in the list by Latin numbers in bold.

I: Arkhangai Aimag, Tsenkher Somon, between Tsetserleg and Tsenkher settlements, Urd-Tamir River valley, 4704'18'N, 10059'25'E, elev. $2100 \mathrm{~m}$, Larix sibirica forest with stones.

II: Arkhangai Aimag, Bulgan Somon, Khul Sayayn Davaa pass, 4649'52"N, 10048'45"E, elev. $2800 \mathrm{~m}$, mountain tundra.

III: Khovd Aimag, Altai Somon, near Barlag settlement, 4553'42”N, 9312'24”E, elev. 1850 m, steppe with shale rock outcrops. 
IV: Khovd Aimag, Must Somon, Bodonch Gol River valley, 46³1'53”N, 92²3'28'E, elev. 2400 $\mathrm{m}$, sandstone boulders in steppe.

v: Khovd Aimag, Must Somon, Bodonch Gol River valley, Mt. Zurkh Uul, 46 $30^{\prime} 44^{\prime \prime} \mathrm{N}$, $92^{\circ} 20^{\prime} 41^{\prime \prime} E$, elev. 2600-3000 m, mountain tundra with stones and rocks.

VI: Khovd Aimag, Must Somon, Baga Ulaan Davaa pass, 4641'38'N, 92¹7'38'E, elev. 3000 $\mathrm{m}$, steppe with sandstone boulders.

VII: Khovd Aimag, Mankhan Somon, $30 \mathrm{~km} \mathrm{SW}$ of Mankhan settlement, Khoid Tsenkher Gol

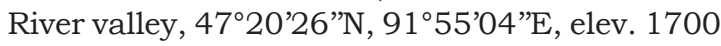
$\mathrm{m}$, steppe with shale rocks and stones.

VIII: Bayan-Ulgii Aimag, Tolbo Somon, 45 km E of Tolbo settlement, Khashaatyn Davaa pass, 48³0'30"N, 9042'57'E, elev. 2630 m, steppe with shale rocks and stones.

IX: Bayan-Ulgii Aimag, Tolbo Somon, $15 \mathrm{~km} \mathrm{E}$ of Tolbo settlement, Mt. Sairyn Uul, 48 22'39'N, 90²9'29'E, elev. $3050 \mathrm{~m}$, mountain tundra with stones.

X: Bayan-Ulgii Aimag, Tolbo Somon, $15 \mathrm{~km} \mathrm{E}$ of Tolbo settlement, Mt. Sairyn Uul, $48^{\circ} 21^{\prime} 48^{\prime \prime N}$, $90^{\circ} 29^{\prime} 87^{\prime \prime} \mathrm{E}$, elev. 2600-2800 m, steppe and rocks on mountain slope.

XI: Bayan-Ulgii Aimag, Tsengel Somon, WNW of Zagastnuur settlement, Tsagaan Gol River valley, in the vicinities of Potanina glacier, 4904'41'N, 8805'16”E, elev. 2600 m, steppe.

XII: Bayan-Ulgii Aimag, Tsengel Somon, WNW of Zagastnuur settlement, Tsagaan Gol River valley, in the vicinities of Potanina glacier,

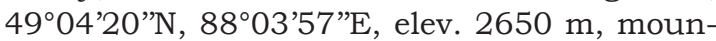
tain tundra with Betula rotundifolia shrubs.

XIII: Zavkhan Aimag, Ikh-Uul Somon, Solongotyn Davaa pass, 48 $17^{\prime} 26^{\prime \prime} \mathrm{N}, 98^{\circ} 57^{\prime} 31^{\prime \prime} \mathrm{E}$, elev. 2600 m, wet mountain tundra with Betula rotundifolia shrubs.

XIV: Arkhangai Aimag, Tsakhir Somon, near Tsagannuur settlement, $48^{\circ} 04^{\prime} 30^{\prime \prime} \mathrm{N}$, 99²5'33"E, elev. 2060 m, steppe.

XV: Arkhangai Aimag, Taryat Somon, NW of Taryat settlement, near Khorgo Uul volcano, $48^{\circ} 11^{\prime} 28^{\prime \prime} \mathrm{N}, 99^{\circ} 49^{\prime} 47^{\prime \prime} \mathrm{E}$, elev. $2080 \mathrm{~m}$, volcanic stone field with sparse Larix sibirica trees.

\section{THE SPECIES}

Taxa newly reported for Asia are denoted by '‘*', those new to Mongolia by '*'. Lichenicolous lichens are designated by 'L'.

ABrothallus CAERULESCEnS I. Kotte All on thalli of Xanthoparmelia stenophylla. III: 13.07.2019, O. Enkhtuya (LE 309977); XV: 27.07.2019, M. P. Zhurbenko 19287 (LE 309979); 27.07.2019, M. P. Zhurbenko 19295c (LE 309978). - New to Arkhangai and Khovd Aimags of Mongolia.

ABRothallus PARMELIARUm (Sommerf.) Arnold I: on Parmelia omphalodes (thallus), 8.07.2019, M. P. Zhurbenko 19311a (LE 309999a); II: on P. saxatilis (thallus), 9.07.2019, O. Enkhtuya (LE 310000a). - New to Arkhangai Aimag of Mongolia.

ARthonia Clemens (Tul.) Th. Fr.

All on apothecia of Rhizoplaca chrysoleuca. II: 9.07.2019, M. P. Zhurbenko 19324a (LE 310065); IV: 14.07.2019, M. P. Zhurbenko 19351 (LE 310063); VI: 16.07.2019, M. P. Zhurbenko 19303 (LE 310061); VII: 17.07.2019, O. Enkhtuya (LE 310062); XV: 27.07.2019, M. P. Zhurbenko 19288 (LE 310064). - New to Arkhangai and Khovd Aimags of Mongolia.

*ARTHONIA EPIPHYSCIA Nyl.

XI: on Physcia caesia (thallus), 22.07.2019, O. Enkhtuya (LE 309998). - A common lichenicolous ascomycete with subcosmopolitan distribution (Brackel, 2014).

*ARthonia EXCEntrica Th. Fr.

Both on thalli of Lepraria neglecta agg. IX: 19.07.2019, M. P. Zhurbenko 19299 (LE 309990); XI: 22.07.2019, O. Enkhtuya (LE 309989). - In our material the ascospores are larger than was reported in the protologue (Fries, 1867), viz. (12.1-)13.9-16.7(-18.5) $\times$ (5.2-)5.6-6.6(-7.6) $\mu \mathrm{m}, \mathrm{L} / \mathrm{B}=(2.1-) 2.2-2.8(-3.1)$ $(\mathrm{n}=34)$ vs. $11-13 \times 4-5 \mu \mathrm{m}$. Kowalewska $\&$ Kukwa (2003) also reported for this species ascospores size similar to ours, viz. 13-16 $\times$ 5-6.5 $\mu \mathrm{m}$. Known from scattered finds in Europe, Asia, North America and South America, mainly in the arctic-oreophytic environments (Alstrup \& Hawksworth, 1990; Hafellner \& Türk, 1995; Santesson, 1998; Diederich \& Sérusiaux, 2000; Hafellner et al., 2002; Santesson et al., 
2004; Etayo \& Sancho, 2008; Hafellner, 2008; Zhurbenko 2009a; Etayo, 2010).

*ARthonia molendoi (Frauenf.) R. Sant. All on apothecia and thalli of Rusavskia elegans. IV: 14.07.2019, M. P. Zhurbenko 19353a (LE 310014a); V: 15.07.2019, O. Enkhtuya (LE 310017b); VIII: 18.07.2019, M. P. Zhurbenko 19300 (LE 310015). - A common lichenicolous ascomycete with subcosmopolitan distribution (Alstrup \& Cole, 1998; Alstrup \& Hawksworth, 1990; Etayo \& Sancho, 2008; Hafellner \& Muggia, 2006; Øvstedal \& Lewis Smith, 2001; Zhurbenko, 2009b).

**ARTHONIA XANTHOPARMELIARUM Etayo

XV: on Xanthoparmelia stenophylla (thallus), 27.07.2019, M. P. Zhurbenko 19282b (LE 309973b). - This species was described from South America (Chile: Etayo \& Sancho, 2008) and subsequently also reported from North America (the U.S.A.: Kocourková, 2009) and Europe (Luxembourg: Eichler et al., 2010).

Bachmanniomyces punctum (A. Massal.) Diederich \& Pino-Bodas

I: on Cladonia amaurocraea (podetia), 8.07.2019, M. P. Zhurbenko 19308 (LE 310021); XIII: on C. arbuscula (podetia), 26.07.2019, M. P. Zhurbenko 19355 (LE 310020); on C. amaurocraea (podetia), 26.07.2019, M. P. Zhurbenko 19339 (LE 310022). - New to Arkhangai and Zavkhan Aimags of Mongolia.

\section{${ }^{\mathrm{L}}$ CALOPLACA EPITHALLINA Lynge}

II: on Rhizoplaca chrysoleuca (thallus), 9.07.2019, M. P. Zhurbenko 19362 (LE 310068); VII: on R. chrysoleuca (thallus), 17.07.2019, O. Enkhtuya (LE 310067); IX: on Psorinia conglomerata (thallus), 19.07.2019, M. P. Zhurbenko 19280 (LE 309966). - A rather common lichenicolous lichen widely distributed in the arctic-oreophytic environments of the Holarctic, including Mongolia (Poelt, 1985).

\section{*CARbonea vitellinaria (Nyl.) Hertel}

IX: on Candelariella sp. (thallus), 19.07.2019, M. P. Zhurbenko 19281 (LE 310010). - A common lichenicolous ascomycete with cosmopolitan distribution (Brackel, 2014).

Cercidospora cf. macrospora (Uloth) Hafellner \& Nav.-Ros.
$\mathbf{X}$ : on saxicolous Lecanora sp. (thallus), 19.07.2019, S. Javkhlan (LE 310152); XV: on Rhizoplaca subdiscrepans (thallus), 27.07.2019, S. Javkhlan (LE 310151). - The material examined differs from the species description in Calatayud et al. (2013) in its sometimes strongly heteropolar, somewhat longer and wider ascospores, (21.0-)23.9-29.3(-30.9) × (5.6-)6.0-7.0(-7.5) $\mu \mathrm{m}$, $\mathrm{L} / \mathrm{B}=(3.1-) 3.7-4.5(-4.8)(\mathrm{n}=18)$ vs. $(19-) 20-$ $25(-30) \times 4-6(-7) \mu \mathrm{m}, \mathrm{L} / \mathrm{B}=(3.0-) 3.8-5.4(-6.7)$ (Calatayud et al., 2013). It is also similar to $C$. crozalsiana (H. Olivier) Nav.-Ros., Cl. Roux \& Casares, growing on Squamarina, which differs in somewhat longer ascospores, (22-)24.5-32($37) \times(5-) 5.5-7(-8) \mu m, \mathrm{~L} / \mathrm{B}=(3.2-) 3.7-5.5(-6.8)$ (Calatayud et al., 2013).

Cercidospora verrucosaria (Linds.) Arnold II: on Megaspora verrucosa (thallus, thalline margins of apothecia), 9.07.2019, M. P. Zhurbenko 19328 (LE 309954). - New to Arkhangai Aimag of Mongolia.

${ }^{*}$ Cercidospora xanthoriae (Wedd.) R. Sant. All on apothecia and thalli of Rusavskia elegans. III: 13.07.2019, O. Enkhtuya (LE 310016); IV: 14.07 .2019 , M. P. Zhurbenko 19353c (LE 310013); V: 15.07.2019, O. Enkhtuya (LE 310017a); X: 19.07.2019, O. Enkhtuya (LE 310018). - Known from scattered finds in Europe, Asia and North America (Alstrup \& Hawksworth, 1990; Sérusiaux et al., 1999; Hafellner, 2002, 2018; Santesson et al., 2004; Knudsen \& Lendemer, 2006; Zhurbenko, 2009b; Joshi et al., 2016).

\section{Clypeococcum cetrariae Hafellner}

II: on Cetraria laevigata (thallus), 9.07.2019, M. P. Zhurbenko 19323b (LE 310047); on Flavocetraria cucullata (thallus), 9.07.2019, E. Enkhtaivan (LE 310044); XI: on F. cucullata (thallus), 22.07.2019, O. Enkhtuya (LE 310046) XIII: on Cetraria laevigata (thallus), 26.07.2019, M. P. Zhurbenko 19335 (LE 310045). - New to Arkhangai, Bayan-Ulgii and Zavkhan Aimags of Mongolia.

Corticifraga Peltigerae (Fuckel) D. Hawksw. \& R. Sant.

XIII: on adjacent thalli of Peltigera extenuata and P. rufescens, 26.07.2019, M. P. Zhurbenko 19338 (LE 309965). - New to Zavkhan Aimag of Mongolia. 
Didymocyrtis CLADONiICola (Diederich, Kocourk. \& Etayo) Ertz \& Diederich

XIII: on Cladonia amaurocraea (podetia), 26.07.2019, M. P. Zhurbenko 19344 (LE 310027); XV: on C. gracilis (podetia), 27.07.2019, M. P. Zhurbenko 19291 (LE 310026). - New to Arkhangai and Zavkhan Aimags of Mongolia.

DIDYMOCYRTIS CONSIMILIS Vain.

V: on Caloplaca cerina (apothecia), 15.07.2019, O. Enkhtuya (LE 310004). - New to Khovd Aimag of Mongolia.

*Didymocyrtis RHizoplacae Y. Joshi \& K. Bisht XV: on Rhizoplaca chrysoleuca (apothecia), 27.07.2019, M. P. Zhurbenko 19363 (LE 310069). - The material examined differs from the species protologue (Joshi et al., 2018) in 1 (rarely 2-3)-septate vs. 1-septate, larger ascospores, (7.5-)10.2-14.2(-17.0) × (4.7-)5.0$6.0(-6.6) \mu \mathrm{m}$ vs. $10-12 \times 4-5 \mu \mathrm{m}$, and orbicular, broadly oblong or broadly ellipsoid vs. ellipsoid, larger conidia, (4.1-)4.9-7.3(-9.2) × (3.7-)4.1$5.3(-6.5) \mu \mathrm{m}$ vs. $(4-) 5 \times 3 \mu \mathrm{m}$. This species has been reported from Mongolia as Didymocyrtis sp. in Zhurbenko et al. (2019). Previously it was known only from India (Joshi et al., 2018).

\section{ECHINOTHECIUM HYPOGYMNIAE Zhurb.}

II: on Hypogymnia austerodes (thallus), 9.07.2019, O. Enkhtuya (LE 309970). - New to Arkhangai Aimag of Mongolia.

\section{ECHINOTHECIUM RETICULATUM Zopf}

II: on Parmelia omphalodes (thallus), 9.07.2019, O. Enkhtuya (LE 310000b); IX: on $P$. saxatilis (thallus), 19.07.2019, M. P. Zhurbenko 19272 (LE 310001). - New to Arkhangai and BayanUlgii Aimags of Mongolia.

Endococcus cf. MACROSPORUS (Arnold) Nyl.

X: on Rhizocarpon sp. (thallus), 19.07.2019, O. Enkhtuya (LE 310137). - Ascomata 120-160 $\mu \mathrm{m}$ diam., mostly only slightly protruding, occasionally half erumpent, up to several tens per host areole, aggregated, host lobes sometimes slightly swollen and bleached under heavy infections. Ascospores pale brownish grey to finally medium brown, ellipsoid to narrowly ellipsoid, with rather acute apices, 1-septate, more or less homopolar, sometimes constricted at the septum, (14.7-)16.3-19.5(-22.7) × 6.2)6.9-8.1(-8.9) $\mu \mathrm{m}, \mathrm{L} / \mathrm{B}=(1.8-) 2.1-2.7(-3.1)(\mathrm{n}$ $=55)$, wall smooth, sometimes apically slightly darker, guttulate, particularly when immature. According to Sérusiaux et al. (1999) Endococcus macrosporus is characterized by somewhat narrower ascospores, $16.5-19.5 \times 5.5-7 \mu \mathrm{m}$. The material examined is also similar to Endococcus sardous Brackel, however, this species differs in having one or few ascomata per host areole, not inducing swellings of the host thallus, and often slightly heteropolar, somewhat narrower and more elongated ascospores, (14.0-)16.7$20.8(-23.0) \times(5.0-) 5.8-7.0 \mu \mathrm{m}, \mathrm{L} / \mathrm{B}=(2.1-) 2.5-$ $3.4(-4.6)$, not constricted at the septum, without darkening of the apical wall (Brackel \& Berger, 2019). So far Endococcus macrosporus has not been reported from Mongolia.

*Endococcus propinquUs (Körb.) D. Hawksw. s. lat.

II: on a saxicolous crustose lichen (thallus), 9.07.2019, M. P. Zhurbenko 19331 (LE 310139). - A common lichenicolous ascomycete with cosmopolitan distribution (Brackel, 2014).

ENDOCOCCUS cf. RUGULOSUs (Leight.) Nyl. s. lat. v: on Rhizocarpon disporum (thallus), 15.07.2019, O. Enkhtuya (LE 310138). - Ascomata 150-250 $\mu \mathrm{m}$ diam., mainly semi-immersed. Ascospores medium brown, homopolar, ends rounded, 1-septate, constricted at the septum, (12.3-)13.3-14.9(-15.2) × (7.1-)7.7-8.9(-9.6) $\mu \mathrm{m}$, $\mathrm{L} / \mathrm{B}=(1.5-) 1.6-1.8(-2.0)(\mathrm{n}=25)$. The material examined corresponds to the broad species concept of Endococcus rugulosus presented by Triebel (1989), with the exception of somewhat narrower ascospores, (12-)13-16(-16.5) $\times$ (5.5-)6-7.5(-8) $\mu \mathrm{m}$, cited by this author. So far Endococcus rugulosus has not been reported from Mongolia.

*Intralichen Christiansenil (D. Hawksw.) D. Hawksw. \& M.S. Cole

IV: on Rusavskia elegans (apothecia), 14.07.2019, M. P. Zhurbenko 19353b (LE 310014b); IX: on Candelariella canadensis (apothecia), 19.07.2019, M. P. Zhurbenko 19265 (LE 310012). - A common lichenicolous hyphomycete with cosmopolitan distribution (Brackel, 2014).

**KATHERINOMYCES CETRARIAE Khodos. s. lat.

II: on Cetraria laevigata (thallus), 9.07.2019, M. P. Zhurbenko 19323c (LE 310049); on Rhizoplaca chrysoleuca (apothecia), 9.07.2019, M. P. Zhurbenko 19324c (LE 310054); on Fla- 
vocetraria cucullata (thallus), 9.07.2019, M. P. Zhurbenko 19329a (LE 310051); IV: on $R$. chrysoleuca (apothecia, thallus), 14.07.2019, M. P. Zhurbenko 19357 (LE 310053); IX: on Flavocetraria nivalis (thallus), 19.07.2019, M. P. Zhurbenko 19268b (LE 310052); XI: on Cetraria islandica (thallus), 22.07.2019, M. P. Zhurbenko 19313 (LE 310050); XIII: on F. cucullata (thallus), 26.07.2019, M. P. Zhurbenko 19333a (LE 310048a). - The specimens examined differ from the species protologue (Khodosovtsev et al., 2016) in having 0(-1)-septate vs. aseptate, larger and less elongated conidia (Fig. 1), (5.2-)7.5-11.5(-17.2) × (3.4-)5.1-6.7(-7.6) $\mu \mathrm{m}, \mathrm{L} / \mathrm{B}=(1.0-) 1.3-1.9(-3.0)(\mathrm{n}=282) \mathrm{vs}$. (4.3-)6.7-10.5(-16.3) × (2.8-)3.5-4.7(-6.0) $\mu \mathrm{m}$, $\mathrm{L} / \mathrm{B}=(1.1-) 1.5-2.7(-5.0)$, and in inducing strong discoloration of the host lobes. Shape and size of conidia significantly vary in different specimens, even on the same host species. Vegetative hyphae are usually macroscopically conspicuous as a dense net of tiny dark hyphae. So far this species was known only from Ukraine, growing on Cetraria aculeata (type host) and Lecidea fuscoatra (Khodosovtsev et al., 2016; Darmostuk $\&$ Khodosovtsev, 2019), hence Flavocetraria and Rhizoplaca are new host genera.

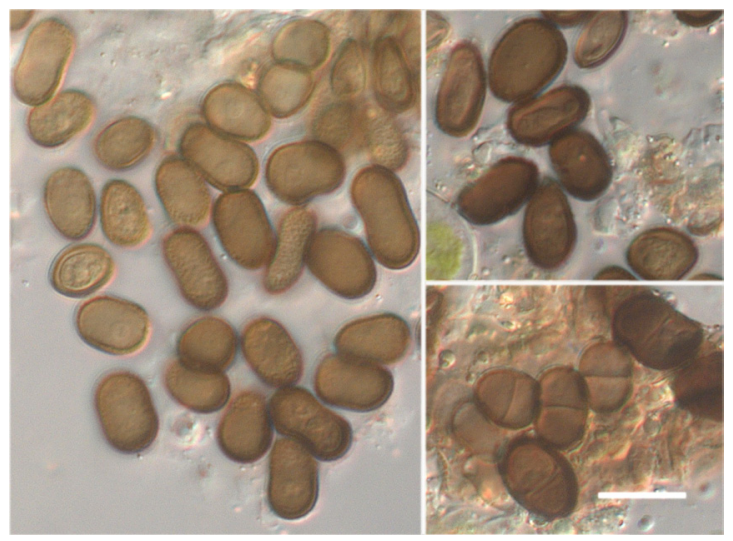

Fig. 1. Conidia of Katherinomyces cetrariae s. lat. growing on Rhizoplaca chrysoleuca (LE 310053; in water). Scale bar $=10 \mu \mathrm{m}$.

**LICHENOCHORA ARCTICA Zhurb.

VII: on Candelariella aggregata (growing on the prothallus between areoles and apothecia), 17.07.2019, O. Enkhtuya (LE 310008). - Formerly known only from the Canadian Arctic (Zhurbenko, 2013b).
*Lichenochora CALOPlacae Zhurb.

II: on Athallia saxifragarum (thallus), 9.07.2019, M. P. Zhurbenko 19322 (LE 310005). - Formerly known from Norway (Svalbard), Russia (the Caucasus and Severnaya Zemlya archipelago) and Ukraine (Zhurbenko \& Brackel, 2013; Khodosovtsev \& Darmostuk, 2017; Zhurbenko, 2017).

*Lichenochora Rinodinae Zhurb.

IX: on Rinodina mniaraea (thallus), 19.07.2019, M. P. Zhurbenko 19273 (LE 309959). - Formerly known from the arctic parts of Canada, Norway (Svalbard), Russia and the U.S.A., as well as from the Russian Caucasus (Zhurbenko, 2013b; Zhurbenko \& Kobzeva, 2014).

Lichenoconium erodens M.S. Christ. \& D. Hawksw.

II: on Cetraria laevigata (thallus), 9.07.2019, M. P. Zhurbenko 19323d (LE 310041); V: on Vulpicida juniperinus (thallus), 15.07.2019, O. Enkhtuya (LE 310042); IX: on V. juniperinus (thallus), 19.07.2019, M. P. Zhurbenko 19278 (LE 310040). - New to Arkhangai and BayanUlgii Aimags of Mongolia.

Lichenoconium lecanorae (Jaap) D. Hawksw.

All on apothecia, occasionally on thalli of Rhizoplaca chrysoleuca. II: 9.07.2019, M. P. Zhurbenko 19324b (LE 310074); VI: 16.07.2019, M. P. Zhurbenko 19368 (LE 310073); VII: 17.07.2019, O. Enkhtuya (LE 310071); VIII: 18.07.2019, M. P. Zhurbenko 19317b (LE 310072); XV: 27.07.2019, M. P. Zhurbenko 19368 (LE 310070). - New to Arkhangai, Bayan-Ulgii and Khovd Aimags of Mongolia.

Lichenoconium pyxidatae (Oudem.) Petr. \& Syd. V: on Cladonia pyxidata (basal squamules), 15.07.2019, O. Enkhtuya (LE 310025). - New to Khovd Aimag of Mongolia.

Lichenoconium usneae (Anzi) D. Hawksw.

IX: on Evernia terrestris (thallus), 19.07.2019, M. P. Zhurbenko 19270 (LE 309969); on Flavocetraria nivalis (thallus), 19.07.2019, M. P. Zhurbenko 19268a (LE 310039). - New to Bayan-Ulgii Aimag of Mongolia.

*Lichenodiplis lecanorae (Vouaux) Dyko \& D. Hawksw.

V: on Athallia saxifragarum (apothecia), 15.07.2019, O. Enkhtuya (LE 310006). - A common lichenicolous coelomycete with cosmopolitan distribution (Brackel, 2014). 
Lichenopeltella Cetrariae (Bres.) Höhn.

II: on Cetraria laevigata (thallus), 9.07.2019, E. Enkhtaivan (LE 310038). - New to Arkhangai Aimag of Mongolia.

Lichenopeltella Cladoniarum E.S. Hansen \& Alstrup

II: on Cladonia arbuscula (podetia), 9.07.2019, E. Enkhtaivan (LE 310024). - New to Arkhangai Aimag of Mongolia.

Lichenostigma Alpinum (R. Sant., Alstrup \& D. Hawksw.) Ertz \& Diederich

II: on Ochrolechia frigida (apothecia, thallus), 9.07.2019, O. Enkhtuya (LE 309971a); on Flavocetraria cucullata (thallus), 9.07.2019, M. P. Zhurbenko 19329c (LE 310043); V: on Cladonia pocillum (basal squamules, cups), 15.07.2019, O. Enkhtuya (LE 310023b). -

Mostly confined to Lepra, Ochrolechia and Varicellaria (Diederich et al., 2018), but was also reported from many other lichen genera (Brackel, 2014). New to Arkhangai and Khovd Aimags of Mongolia.

LiCHENOSTIGMA CHLAROTERAE (F. Berger \& Brackel) Ertz \& Diederich

$\mathbf{X}$ : on Lecanora sp. growing on fine earth deposits with plant remnants on rock (apothecia, thallus), 19.07.2019, O. Enkhtuya (LE 310154); on saxicolous Lecanora sp. (thallus), 19.07.2019, S. Javkhlan (LE 310155). - New to Bayan-Ulgii Aimag of Mongolia.

Lichenostigma cosmopolites Hafellner \& Calat. All on thalli of Xanthoparmelia stenophylla. I: 8.07.2019, M. P. Zhurbenko 19312 (LE 309974); II: 9.07.2019, O. Enkhtuya (LE 309975); XV: 27.07.2019, M. P. Zhurbenko 19295b (LE 309976b). - New to Arkhangai Aimag of Mongolia.

*Lichenostigma dimelaenae Calat. \& Hafellner

X: on Dimelaena oreina (thallus), 19.07.2019, S. Javkhlan (LE 310164). - Previously known from scattered finds in Europe (Ukraine: Darmostuk et al., 2018), Asia (Turkey: Yazici \& Etayo 2014) and North America (Arizona and California in the U.S.A.: Calatayud et al., 2004; Kocourková $\&$ Knudsen, 2015).

LiCHENOSTIGMA MAURERI Hafellner

IX: on Evernia terrestris (thallus), 19.07.2019, M. P. Zhurbenko 19271 (LE 309968); XV: on Ramalina pollinaria (thallus), 27.07.2019, M.
P. Zhurbenko 19289 (LE 309953). - New to Arkhangai and Bayan-Ulgii Aimags of Mongolia.

Lichenostigma cf. RouxiI Nav.-Ros., Calat. \& Hafellner

III: on Lobothallia alphoplaca (apothecia, thallus), 13.07.2019, O. Enkhtuya (LE 310160); IV: on Aspicilia sp. (thallus), 14.07.2019, M. P. Zhurbenko 19349a (LE 310163); X: on L. alphoplaca (apothecia, thallus), 19.07.2019, S. Javkhlan (LE 310158); on Lecanora baicalensis (thallus, occasionally apothecia), 19.07.2019, O. Enkhtuya (LE 310161). - The identification is somewhat uncertain because according to Calatayud et al. (2002) this species is confined to Squamarina; so far it has not been reported from Mongolia.

*Lichenostigma triseptatum Halic1 \& D. Hawksw. V: on Aspicilia sp. (thallus), 15.07.2019, O. Enkhtuya (LE 310162). - Previously known from scattered finds in Asia (India, Iran and Turkey: Halici \& Hawksworth, 2007; Valadbeigi \& Sipman, 2010; Yazici \& Etayo, 2014; Joshi et al., 2016).

Lichenothelia RUgosa (G. Thor) Ertz \& Diederich II: on Diploschistes muscorum (thallus, occasionally apothecia), 9.07.2019, O. Enkhtuya (LE 309984); X: on D. scruposus (thallus), 19.07.2019, O. Enkhtuya (LE 309983); XV: on D. scruposus (thallus, occasionally apothecia), 27.07.2019, M. P. Zhurbenko 19286 (LE 309982). - New to Arkhangai and Bayan-Ulgii Aimags of Mongolia.

Muellerella ERRATica (A. Massal.) Hafellner \& V. John

II: on Lecidea lapicida var. pantherina (thallus), 9.07.2019, O. Enkhtuya (LE 310144); IV: on L. lapicida (thallus), 14.07.2019, S. Javkhlan (LE 310143); on saxicolous Verrucaria sp. (thallus), 14.07.2019, M. P. Zhurbenko 19347 (LE 310141); on a saxicolous crustose lichen (thallus), 14.07.2019, M. P. Zhurbenko 19346b (LE 310140b); X: on Lecidea lapicida (thallus), 19.07.2019, O. Enkhtuya \& S. Javkhlan (LE 310147. - Verrucaria is a rarely reported host genus for this species (Brackel, 2014; Knudsen $\&$ Kocourkova, 2009). New to Arkhangai, BayanUlgii and Khovd Aimags of Mongolia.

Muellerella lichenicola (Sommerf.) D. Hawksw. II: on muscicolous sorediate Biatora sp. (thal- 
lus), 9.07.2019, O. Enkhtuya (LE 310142a); III: on Aspicilia sp. (thallus), 13.07.2019, O. Enkhtuya (LE 310146). - New to Arkhangai and Khovd Aimags of Mongolia.

Muellerella pygmaea (Körb.) D. Hawksw. s. str. V: on Acarospora sp. (thallus), 15.07.2019, O. Enkhtuya (LE 310145); VI: on Rusavskia elegans (thallus), 16.07.2019, M. P. Zhurbenko 19302 (LE 310003); VIII: on R. elegans (thallus), 18.07.2019, M. P. Zhurbenko 19318 (LE 310019); XV: on Anamylopsora pulcherrima (thallus), 27.07.2019, M. P. Zhurbenko 19290a (LE 309967). - New to Arkhangai, Bayan-Ulgii and Khovd Aimags of Mongolia.

Muellerella cf. ventosicola (Mudd) D. Hawksw. IV: on Aspicilia sp. (thallus), 14.07.2019, M. P. Zhurbenko 19349b (LE 310135); VIII: on Aspicilia sp. (thallus), 18.07.2019, M. P. Zhurbenko 19320a (LE 310134a). - Ascospores are somewhat larger than was reported by Triebel (1989), viz. (6.2-)7.3-9.3(-10.3) × (4.2-)4.8-5.6(-6.3) $\mu \mathrm{m}$, $\mathrm{L} / \mathrm{B}=(1.2-) 1.4-1.8(-2.0)(\mathrm{n}=62)$ vs. $6.5-8.5(-9)$ $\times 4-5.5(-6) \mu \mathrm{m}$. This species was described from Ophioparma ventosa (Mudd, 1861) and according to Triebel (1989) also grows on species of Dimelaena, Protoparmelia and Rhizocarpon. However, it has been already reported from Aspicilia (Kukwa \& Flakus, 2009) and Lecanora (Etayo, 2010). It is characterized by subcosmopolitan distribution (Brackel, 2014), but so far has not been reported from Mongolia.

NESOleChiA OXYsPoRA (Tul.) A. Massal. var. FUSCA (Triebel \& Rambold) Diederich

II: on Xanthoparmelia stenophylla (thallus), 9.07.2019, O. Enkhtuya (LE 309981); XV: on X. conspersa (thallus), 27.07.2019, M. P. Zhurbenko 19374 (LE 310168); on X. stenophylla (thallus), 27.07.2019, M. P. Zhurbenko 19295a (LE 309980). - New to Arkhangai Aimag of Mongolia.

NESOlechia OXYSPORA (Tul.) A. Massal. var. OXYSPORA

Both on thalli of Parmelia omphalodes. I: 8.07.2019, M. P. Zhurbenko 19311b (LE 309999b); XV: 27.07.2019, M. P. Zhurbenko 19294a (LE 310002a). - New to Arkhangai Aimag of Mongolia.

*Niesslia Peltigericola (D. Hawksw.) Etayo I: on Peltigera aphthosa (moribund parts of thallus), 8.07.2019, M. P. Zhurbenko 19309b
(LE 309963b). - Known from scattered finds in Europe, Asia and North America (Hawksworth, 1980; Alstrup \& Hawksworth, 1990; Hafellner \& Türk, 1995; Alstrup \& Cole, 1998; Diederich, 2003; Santesson et al., 2004; Sohrabi \& Alstrup, 2007; Kukwa \& Flakus 2009; Zhurbenko, 2009b).

*Polycoccum trypethelioides (Th. Fr.) R. Sant. XV: on Stereocaulon sp. (thallus), 27.07.2019, S. Javkhlan (LE 309956). - Widely distributed in the Holarctic (Hafellner \& Türk, 1995; Berger, 2000; Zhurbenko, 2010), but is also known from South America (Etayo \& Sancho, 2008; Zhurbenko \& Ohmura, 2019).

Polycoccum vermicularium (Linds.) D. Hawksw. All on thalli of Thamnolia vermicularis $(\mathrm{K}+$ pale yellow). XI: 22.07.2019, M. P. Zhurbenko 19314 (LE 309987); XII: 22.07.2019, M. P. Zhurbenko 19297 (LE 309988); XIII: 26.07.2019, M. P. Zhurbenko 19334 (LE 309986). - New to BayanUlgii and Zavkhan Aimags of Mongolia.

PRONECTRIA ERYTHRINELLA (Nyl.) Lowen

XIII: on adjacent thalli of Peltigera extenuata (mainly) and P. rufescens, 26.07.2019, M. P. Zhurbenko 19342 (LE 309961). - New to Zavkhan Aimag of Mongolia.

Pyrenidium aCtinellum Nyl. s. lat.

XV: on Diploschistes scruposus (thallus), 27.07.2019, O. Enkhtuya (LE 309985). - New to Arkhangai Aimag of Mongolia.

*Rhymbocarpus neglectus (Vain.) Diederich \& Etayo

Both on thalli of Lepraria neglecta agg. IX: 19.07.2019, M. P. Zhurbenko 19285 (LE 309991); XI: 22.07.2019, O. Enkhtuya (LE 309992). - Widely distributed in the Holarctic (Kümmerling et al., 1993; Diederich \& Etayo, 2000).

Rosellinula fRUstulosae (Vouaux) R. Sant.

VII: on Lecanora argopholis (thallus), 17.07.2019, O. Enkhtuya (LE 310149). - New to Khovd Aimag of Mongolia.

${ }^{\text {LSARCOGYNE SPHAEOSPORA J. Steiner }}$

All on thalli of sterile Candelariella species growing on boulders or on fine earth deposits on boulders. IV: 14.07.2019, M. P. Zhurbenko 19352 (LE 310007); VI: 16.07.2019, M. P. Zhur- 
benko 19305 (LE 310009); VIII: 18.07.2019, M. P. Zhurbenko 19319 (LE 310011); M. P. Zhurbenko 19320b (LE 310134b). - This scarcely lichenized lichenicolous fungus growing on Candelariella is so far known from scattered finds in Asia (India, Mongolia, Tajikistan and Turkey: Huneck et al., 1992; Kudratov \& Mayrhofer, 2002; Zhurbenko, 2013a) and North America (California in the U.S.A.: Lendemer et al., 2009).

Sclerococcum Deminutum (Th.Fr.) Ertz \& Diederich

IX: on a muscicolous crustose lichen (thallus), 19.07.2019, M. P. Zhurbenko 19373 (LE 310148); XIII: on Psoroma hypnorum (apothecia, thallus), 26.07.2019, M. P. Zhurbenko 19335 (LE 309955). - New to Bayan-Ulgii and Zavkhan Aimags of Mongolia.

SphaERELlothecium ARANeOSUm (Arnold) Zopf II: on Ochrolechia frigida (apothecia, thallus), 9.07.2019, O. Enkhtuya (LE 309971b). - New to Arkhangai Aimag of Mongolia.

SPHAERELlothecium Cladoniae (Alstrup \& Zhurb.) Hafellner

Both on basal squamules of Cladonia pyxidata. II: 9.07.2019, M. P. Zhurbenko 19330 (LE 310031); IX: 19.07.2019, M. P. Zhurbenko 19283 (LE 310029). - New to Arkhangai and Bayan-Ulgii Aimags of Mongolia.

Sphaerellothecium Cladonilcola E. S. Hansen \& Alstrup

II: on Cladonia arbuscula (podetia), 9.07.2019, M. P. Zhurbenko 19356 (LE 310032); on C. stellaris (podetia), 9.07.2019, O. Enkhtuya (LE 310028); XIII: on C. arbuscula (podetia), 26.07.2019, M. P. Zhurbenko 19340 (LE 310033); XV: on C. rangiferina (podetia), 27.07.2019, S. Javkhlan (LE 310030). - New to Arkhangai and Zavkhan Aimags of Mongolia.

Sphaerellothecium cf. Parmeliae Diederich \& Etayo

Both on thalli of Parmelia omphalodes. I: 8.07.2019, M. P. Zhurbenko 19311c (LE 309999c); XV: 27.07.2019, M. P. Zhurbenko 19294b (LE 310002b). - The taxonomy of this fungus and its differences from the species protologue (Etayo \& Diederich, 1998) have been discussed in Zhurbenko \& Zheludeva (2015) and Zhurbenko et al. (2019). New to Arkhangai Aimag of Mongolia.
*Sphaerellothecium Phaeorrhizae Diederich \& Zhurb.

XV: on Phaeorrhiza sp. (thallus), 27.07.2019, M. P. Zhurbenko 19292 (LE 309993). - In our material the ascospores are slightly longer than reported in the protologue (Diederich \& Zhurbenko, 2009), viz. (10.0-)10.8-12.2(-12.7) $\times$ (4.6-)4.9-5.5(-5.9) $\mu \mathrm{m}, \mathrm{L} / \mathrm{B}=(1.9-) 2.1-2.3(-2.6)$ $(\mathrm{n}=44)$ vs. $9-11.5 \times 4-5(-5.5) \mu \mathrm{m}$. Previously known from a few finds in Russian Asia (Diederich \& Zhurbenko, 2009; Zhurbenko, 2009a).

*Sphaerellothecium PRopinquellum (Nyl.) Cl. Roux \& Triebel

XV: on Lecanora albella (apothecia), 27.07.2019, S. Javkhlan (LE 310150). - Widely distributed in the Holarctic (Brackel, 2014).

SPHAEREllothecium PUMilum (Lettau) Nav.-Ros., Cl. Roux \& Hafellner

I: on Physcia phaea (thallus), 8.07.2019, O. Enkhtuya (LE 309997). - New to Arkhangai Aimag of Mongolia.

Stigmidium MicRocarpum Alstrup \& J. C. David II: on Flavocetraria cucullata (thallus), 9.07.2019, E. Enkhtaivan (LE 310037b). - Known from scattered finds in Europe, Asia (China, Japan, Mongolia, Russia) and North America, often in the arctic-oreophytic environments (Alstrup, 1993; Zhurbenko, 2009a; Brackel, 2014; Zhurbenko \& Ohmura, 2019).

\section{Stigmidium PSORAe (Anzi) Hafellner}

Both on thalli of Psorula rufonigra. I: 8.07.2019, M. P. Zhurbenko 19310a (LE 309958a); XV: 27.07.2019, M. P. Zhurbenko 19293a (LE 309957a). - Stigmidium cf. psorae was formerly reported from Khuvsugul Aimag of Mongolia growing on Psora testacea (Zhurbenko et al., 2019). Here we confirm the occurrence of this species in Mongolia.

STIGMidiUm SOlORINARIUM (Vain.) D. Hawksw.

IX: on Solorina octospora (moribund thallus), 19.07.2019, M. P. Zhurbenko 19262 (LE 309960). - New to Bayan-Ulgii Aimag of Mongolia.

*STIGMIDIUM XANTHOPARMELIARUM Hafellner

$\mathbf{X V}$ : on Xanthoparmelia stenophylla (thallus), 27.07.2019, M. P. Zhurbenko 19282a (LE 309973a). - Known from scattered finds in Eu- 
rope (Hafellner, 1994, 1999, 2018; Calatayud \& Triebel, 1999; Hawksworth, 2003; Suija, 2005; Diederich et al., 2012; Brackel, 2014; Naumovych \& Darmostuk, 2015), Asia (India, Iran, Russia, Turkey: Sohrabi \& Alstrup, 2007; Halici et al., 2009; Zhurbenko \& Kobzeva, 2014; Joshi et al., 2016), North America (Kocourkova $\&$ Knudsen 2008), but also in New Zealand (Hafellner \& Mayrhofer, 2007).

*Taeniolella pertusariicola D. Hawksw. \& H. Mayrhofer

II: on Pertusaria bryontha (apothecia, thallus), 9.07.2019, M. P. Zhurbenko 19326 (LE 309962). - Known from many finds in Greenland, Europe and Asia, but also from Australia (Heuchert et al., 2018).

TAEniolella RolfiI Diederich \& Zhurb.

IX: on Cetrariella delisei (thallus), 19.07.2019, M. P. Zhurbenko 19267 (LE 310035); XI: on Cetraria aculeata (thallus), 22.07.2019, O. Enkhtuya (LE 310034). - New to Bayan-Ulgii Aimag of Mongolia.

*Tetramelas phaeophysciae A. Nordin \& Tibell II: on Physcia caesia (thallus), 9.07.2019, O. Enkhtuya (LE 309996). - Known from scattered finds in Greenland, Europe and Asia, mainly in the arctic-oreophytic environments (Nordin \& Tibell, 2005; Zhurbenko, 2009b; Zhurbenko \& Brackel, 2013; Zhurbenko et al., 2016; Zhurbenko, 2017).

Tetramelas pulverulentus (Anzi) A. Nordin \& Tibell

Both on thalli of Physconia muscigena. v: 15.07.2019, O. Enkhtuya (LE 309994); IX: 19.07.2019, M. P. Zhurbenko 19264 (LE 309995). - New to Bayan-Ulgii and Khovd Aimags of Mongolia.

THELOCARPON EPIBOLUM Nyl. var. EPITHALLINUM (Leight.) G. Salisb.

I: on Peltigera aphthosa (moribund parts of thallus), 8.07.2019, M. P. Zhurbenko 19309a (LE 309963a); IX: on Solorina octospora (moribund parts of thallus), 19.07.2019, M. P. Zhurbenko 19275b (LE 309964b). - Facultatively lichenicolous on Arthrorhaphis, Baeomyces, Catapyrenium, Peltigera, Protopannaria and Solorina (Diederich et al., 2018). New to Arkhangai and Bayan-Ulgii Aimags of Mongolia.
TREMATOSPHAERIOPSIS PARMEliana (Jacz.) Elenkin XIV: on Xanthoparmelia vagans (both sides of lobes), 26.07.2019, M. P. Zhurbenko 19307 (LE 309972). - New to Arkhangai Aimag of Mongolia.

\section{ACKNOWLEDGEMENTS}

The authors are grateful to the Russian-Mongolian complex biological expedition of the Russian and Mongolian Academies of Sciences for the support of the field studies in Mongolia in July 2019. We thank Enkhjargal Enkhtaivan for the nice company and help during the field work. Valeriy Darmostuk, Alexander Khodosovtsev and Wolfgang von Brackel are thanked for very useful taxonomical discussions. The study of $\mathrm{M}$. P. Zhurbenko was carried out within the framework of the research project of the Komarov Botanical Institute of the Russian Academy of Sciences "Biodiversity, ecology, structural and functional features of fungi and fungus-like protists" (AAAA-A19-119020890079-6) using equipment of its Core Facility Center "Cell and Molecular Technologies in Plant Science".

\section{REFERENCES}

Alstrup, V. 1993. News on lichens and lichenicolous fungi from the Nordic countries. Graphis Scripta 5: 96-104.

Alstrup, V. \& Cole, M. S. 1998. Lichenicolous fungi of British Columbia. The Bryologist 101: 221-229. https://doi.org/10.1639/00072745(1998) 101[221:LFOBC]2.0.CO;2

Alstrup, V. \& Hawksworth, D. L. 1990. The lichenicolous fungi of Greenland. Meddelelser om Grønland, Bioscience 31: 1-90.

Berger, F. 2000. Beitrag zur Kenntnis der Flechten und lichenicolen Pilze Islands. Acta Botanica Islandica 13: 69-82.

Brackel, W. von 2014. Kommentierter Katalog der flechtenbewohnenden Pilze Bayerns. Bibliotheca Lichenologica 109: 1-476.

Brackel, W. von \& Berger, F. 2019. Lichenicolous fungi from Sardinia (Italy): new records and a first synopsis. Herzogia 32: 444-471. https:// doi.org/10.13158/heia.32.2.2019.444

Calatayud, V. \& Triebel, D. 1999. Stigmidium neofusceliae (Dothideales s.1.), a new lichenicolous fungus from Spain. Nova Hedwigia 69(3-4): 439-448.

Calatayud, V., Navarro-Rosinés, P. \& Hafellner, J. 2002. A synopsis of Lichenostigma subgen. Lichenogramma (Arthoniales), with a key to the species. Mycological Research 106(10):1230-1242. https://doi.org/ 10.1017/S095375620200655X 
Calatayud, V., Hafellner, J. \& Navarro-Rosinés, P. 2004. Lichenostigma. In: Nash III, T. H., Ryan, B. D., Diederich, P. Gries, C. \& Bungartz, F. (eds.): Lichen Flora of the Greater Sonoran Desert Region, Vol. 2. Lichens Unlimited, Arizona State University, Tempe, Arizona. Pp. 664-669.

Calatayud, V., Navarro-Rosinés, P. \& Hafellner, J. 2013. Contributions to a revision of Cercidospora (Dothideales), 2: Species on Lecanora s. 1., Rhizoplaca and Squamarina. Mycosphere 4: 539-557. https://doi.org/10.5943/mycosphere/4/3/8

Darmostuk, V. V. \& Khodosovtsev, A. Y. 2019. Epibryon kondratyukii sp. nov., a new algicolous fungus, and notes on rare lichenicolous fungi collected in Southern Ukraine. Folia Cryptogamica Estonica 56: 109-116. https://doi.org/10.12697/ fce.2019.56.11

Darmostuk, V. V., Khodosovtsev, A. Y., Naumovich, G. O. \& Kharechko, N. V. 2018. Roselliniella lecideae sp. nov. and other interesting lichenicolous fungi from the Northern Black Sea region (Ukraine). Turkish Journal of Botany 42: 354-361. https:/ / doi.org/10.3906/bot-1709-5

Diederich, P. 2003. New species and new records of American lichenicolous fungi. Herzogia 16: 41-90.

Diederich, P. \& Etayo, J. 2000. A synopsis of the genera Skyttea, Llimoniella and Rhymbocarpus (1ichenicolous Ascomycota, Leotiales). The Lichenologist 32: 423-485. https://doi.org/10.1006/ lich. 2000.0290

Diederich, P. \& Sérusiaux, E. 2000. The lichens and lichenicolous fungi of Belgium and Luxembourg. An annotated checklist. Musée National d'Histoire Naturelle, Luxembourg. 207 pp.

Diederich, P. \& Zhurbenko, M. P. 2009. Sphaerellothecium phaeorrhizae and Zwackhiomyces sipmanii spp. nov. on Phaeorrhiza sareptana from northeastern Asia, with a key to the species of Sphaerellothecium. Bibliotheca Lichenologica 99: 113-122.

Diederich, P., Ertz, D., Eichler, M., Cezanne, R., van den Boom, P., Fischer, E., Killmann, D., Van den Broeck, D. \& Sérusiaux, E. 2012. New or interesting lichens and lichenicolous fungi from Belgium, Luxembourg and northern France. XIV. Bulletin de la Société des naturalistes luxembourgeois 113: 95-115.

Diederich, P., Lawrey, J. D. \& Ertz, D. 2018. The 2018 classification and checklist of lichenicolous fungi, with 2000 non-lichenized, obligately lichenicolous taxa. The Bryologist 121: 340-425. https://doi. org/10.1639/0007-2745-121.3.340

Eichler, M., Cezanne, R., Diederich, P., Ertz, D., Van den Broeck, D., van den Boom, P. \& Sérusiaux, E. 2010. New or interesting lichens and lichenicolous fungi from Belgium, Luxembourg and northern France. XIII. Bulletin de la Société des Naturalistes Luxembourgeois 111: 33-46.

Enhbayaryn, R. (ed.). 2004. The thematic dictionary of Mongolian geographical names. Volumes I-VIII. BCI, Ulaanbaatar.
Etayo, J. 2010. Líquenes y hongos liquenícolas de Aragón. Guineana 16: 1-501.

Etayo, J. \& Diederich, P. 1998. Lichenicolous fungi from the western Pyrenees, France and Spain. IV. Ascomycetes. The Lichenologist 30: 103-120. https://doi.org/10.1006/lich.1997.0121

Etayo, J. \& Sancho, L. G. 2008. Hongos liquenícolas del Sur de Sudamérica, especialmente de Isla Navarino (Chile). Bibliotheca Lichenologica 98: 1-302.

Fries, T. M. 1867. Lichenes Spitsbergenses. Kongliga Svenska Vetenskapsakademiens Handlinger. Ser. 2, $7(2): 3-53$.

Hafellner, J. 1994. Über Funde lichenicoler Pilze und Flechten auf Korsika (Frankreich). Bulletin de la Société Linnéenne de Provence 44: 219-234.

Hafellner, J. 1999. Beiträge zu einem Prodromus der lichenicolen Pilze Österreichs und angrenzender Gebiete. IV. Drei neue Arten und weitere bemerkenswerte Funde hauptsächlich in der Steiermark. Linzer Biologische Beitrage 31(1): 507-532.

Hafellner, J. 2002. Bemerkenswerte Funde von Flechten und lichenicolen Pilzen auf makaronesischen Inseln VI. Über einige Neufunde. Fritschiana 36: 11-17.

Hafellner, J. 2008. Additions and corrections to the checklist and bibliography of lichens and lichenicolous fungi of Insular Laurimacaronesia. IV. Fritschiana 64: 1-28.

Hafellner, J. 2018. Noteworthy records of lichenicolous fungi from various countries on the Balkan Peninsula. Herzogia 31: 476-493. https://doi. org/10.13158/heia.31.1.2018.476

Hafellner, J. \& Mayrhofer, H. 2007. A contribution to the knowledge of lichenicolous fungi and lichens occurring in New Zealand. Bibliotheca Lichenologica 95: 225-266.

Hafellner, J. \& Muggia, L. 2006. Über Vorkommen von Caloplaca erodens in der Steiermark (Österreich). Mitteilungen der Naturwissenschaftichen Vereines für Steiermark 135: 33-49.

Hafellner, J. \& Türk, R. 1995. Über Funde lichenicoler Pilze und Flechten im Nationalpark Hohe Tauern (Kärntner Anteil, Österreich). Carinthia II 185/105: 599-635.

Hafellner, J., Triebel, D., Ryan, B. D. \& Nash III, T. H. 2002. On lichenicolous fungi from North America. II. Mycotaxon 84: 293-329.

Halici, M. G. \& Hawksworth, D. L. 2007. Two new species of lichenicolous fungi from Turkey. The Lichenologist 39: 439-443. https://doi. org/10.1017/S0024282907006251

Halici, M. G., Candan, M. \& Türk, A. O. 2009. Notes on some lichenicolous fungi species from Turkey II. Turkish Journal of Botany 33: 389-392.

Hawksworth, D. L. 1980. Notes on some fungi occurring on Peltigera, with a key to accepted species. Transactions of the British Mycological Society 74: 363-386. https://doi.org/10.1016/S00071536(80)80167-7 
Hawksworth, D. L. 2003. The lichenicolous fungi of Great Britain and Ireland: an overview and annotated checklist. The Lichenologist 35: 191-232. https://doi.org/10.1016/S0024-2829(03)000276

Heuchert, B., Braun, U., Diederich, P. \& Ertz, D. 2018. Taxonomic monograph of the genus Taeniolella s. lat. (Ascomycota). Fungal Systematics and Evolution 2: 69-261. https://doi.org/10.3114/ fuse.2018.02.06

Huneck, S., Ahti, T., Cogt, U., Poelt, J. \& Sipman, H. 1992. Zur Verbreitung und Chemie von Flechten der Mongolei. III. Ergebnisse der MongolischDeutschen Biologischen Expedition seit $1962 \mathrm{Nr}$. 217. Nova Hedwigia 54(3-4): 277-308.

Joshi, Y., Falswal, A., Tripathi, M., Upadhyay, S., Bisht, A., Chandra, K., Bajpai, R. \& Upreti, D. K. 2016. One hundred and five species of lichenicolous biota from India: An updated checklist for the country. Mycosphere 7 (3): 268-294. https:/ / doi. org/10.5943/mycosphere/7/3/3

Joshi, Y., Tripathi, M., Bisht, K., Upadhyay, S., Kumar, V., Pal, N., Gaira, A., Pant, S., Rawat, K. S., Bisht, S., Bajpai, R. \& Halda, J. P. 2018. Further contributions to the documentation of lichenicolous fungi from India. Kavaka 50: 26-33.

Khodosovtsev, A. Ye. \& Darmostuk, V. V. 2017. Zwackhiomyces polischukii sp. nov., and other noteworthy lichenicolous fungi from Ukraine. Polish Botanical Journal 62(1): 27-35. https:// doi.org/10.1515/pbj-2017-0006

Khodosovtsev, A.Y., Gavrylenko, L. M. \& Klymenko, V. M. 2016. Katherinomyces cetrariae gen. et sp. nov. (asexual Ascomycota) and Sphaerellothecium aculeatae sp. nov. (Mycosphaerellaceae), new lichenicolous fungi on Cetraria aculeata in Ukraine. Nova Hedwigia 103(1-2): 47-55. https://doi. org/10.1127/nova_hedwigia/2016/0333

Knudsen, K. \& Kocourkova, J. 2009. Lichens, lichenicolous and allied fungi of the Santa Monica Mountains, Part 4: Additions and corrections to the annotated checklist. Opuscula Philolichenum 7: 29-48.

Knudsen, K. \& Lendemer, J. C. 2006. Changes and additions to the North American lichen mycota V. Mycotaxon 95: 309-313.

Kocourková, J. 2009. Observations on the genus $N e$ olamya, with the description of the new species N. xanthoparmeliae (Ascomycota, genera incertae sedis). Opuscula Philolichenum 6: 137-148.

Kocourková, J. \& Knudsen, K. 2008. Four new lichenicolous fungi from North America. Evansia 25(2): 62-64. https://doi.org/10.1639/07479859-25.3.62

Kocourková, J. \& Knudsen, K. 2015. Notes on the California lichen flora 7: more new records. Opuscula Philolichenum 14: 118-120.

Kowalewska, A. \& Kukwa, M. 2003. Additions to the Polish lichen flora. Graphis Scripta 14: 11-17.
Kudratov, I. \& Mayrhofer, H. 2002. Catalogue of the lichenized and lichenicolous fungi of Tajikistan. Herzogia 15: 91-128.

Kukwa, M. \& Flakus, A. 2009. New or interesting records of lichenicolous fungi from Poland VII: species mainly from Tatra Mountains. Herzogia 22: 191-211.

Kümmerling, H., Triebel, D. \& Rambold, G. 1993. Lepraria neglecta and its lichenicolous fungi. Bibliotheca Lichenologica 53: 147-160.

Lendemer, J. C., Kocourková, J. \& Knudsen, K. 2009. Studies in lichen and lichenicolous fungi: more notes on taxa from North America. Mycotaxon 108: 491-497. https://doi.org/10.5248/108.491

Mudd, W. 1861. A manual of British lichens, description of all the species and varieties, five plates, with figures of the spores of one hundred and thirty species, illustrative of the genera. Darlington. 309 pp. https:/ / doi.org/10.5962/bhl.title.153200

Naumovych, A. O. \& Darmostuk, V. V. 2015. Lichenicolous fungi of the valley of Ingulets river (Ukraine). Chornomorski Botanical Journal 11 (4): 512-520. https://doi.org/ 10.14255/2308$9628 / 15.114 / 7$

Nordin, A. \& Tibell, L. 2005. Additional species in Tetramelas. The Lichenologist 37: 491-498. https:/ / doi.org/10.1017/S0024282905015434

Øvstedal, D. O. \& Lewis Smith, R. I. 2001. Lichens of Antarctica and South Goergia. Cambridge, Cambridge University Press. 411 pp.

Poelt, J. 1985. Caloplaca epithallina. Porträt einer parasitischen Flechte. Botanische Jahrbücher für Systematik, Pflanzengeschichte und Pflanzengeographie [Stuttgart] 107(1-4): 457-468.

Santesson, R. 1998. Fungi lichenicoli exsiccati. Fasc. $11 \& 12$ (nos. 251-300). Thunbergia 28: 1-19.

Santesson, R., Moberg, R., Nordin, A., Tønsberg, T. \& Vitikainen, O. 2004. Lichen-forming and lichenicolous fungi of Fennoscandia. Museum of Evolution, Uppsala University. 359 pp.

Sérusiaux, E., Diederich, P., Brand, A. M. \& van den Boom, P. 1999. New or interesting lichens and lichenicolous fungi from Belgium and Luxembourg. VIII. Lejeunia 162: 1-95.

Sohrabi, M. \& Alstrup, V. 2007. Additions to the lichen mycota of Iran from East Azerbaijan Province. Mycotaxon 100: 145-148.

Suija, A. 2005. Lichenicolous fungi and lichens in Estonia I. Ascomycota. Nova Hedwigia 80(12): 247-267. https://doi.org/10.1127/00295035/2005/0080-0247

Triebel, D. 1989. Lecideicole Ascomyceten. Eine Revision der obligat lichenicolen Ascomyceten auf lecideoiden Flechten. Bibliotheca Lichenologica 35: $1-278$.

Valadbeigi, T. \& Sipman, H. J. M. 2010. New records of lichens and lichenicolous fungi from Iran and their biogeographical significance. Mycotaxon 113: 191-194. https://doi.org/10.5248/113.191 
Yazici, K. \& Etayo, J. 2014. Lichenicolous fungi in Iğdır province, Turkey. Acta Botanica Brasilica 28(1): 1-7. https://doi.org/10.1590/S010233062014000100001

Zhurbenko, M. P. 2009a. Lichenicolous fungi and some lichens from the Holarctic. Opuscula Philolichenum 6: 87-120.

Zhurbenko, M. P. 2009b. Lichenicolous fungi and lichens from the Holarctic. Part II. Opuscula Philolichenum 7: 121-186.

Zhurbenko, M. P. 2010. Lichenicolous fungi and lichens growing on Stereocaulon from the Holarctic, with a key to the known species. Opuscula Philolichenum 8: 9-39.

Zhurbenko, M. P. 2013a. A first list of lichenicolous fungi from India. Mycobiota 3: 19-34. https:/ / doi. org/10.12664/mycobiota.2013.03.03

Zhurbenko, M. P. 2013b. Lichenicolous fungi and some allied lichens from the Canadian Arctic. Opuscula Philolichenum 12: 180-197.

Zhurbenko, M. P. 2017. Lichenicolous fungi of the Caucasus: new species, new records and a second synopsis. Opuscula Philolichenum 16: 267-311.

Zhurbenko, M. P. \& Brackel, W. von 2013. Checklist of lichenicolous fungi and lichenicolous lichens of Svalbard, including new species, new records and revisions. Herzogia 26: 323-359. https:/ / doi. org/10.13158/heia.26.2.2013.323

Zhurbenko, M. P. \& Kobzeva, A. A. 2014. Lichenicolous fungi from Northwest Caucasus, Russia. Herzogia 27: 377-396. https://doi.org/10.13158/ heia.27.2.2014.377

Zhurbenko, M. P. \& Ohmura, Y. 2019. New and interesting records of lichenicolous fungi from the TNS herbarium: Part I. Opuscula Philolichenum 18: 74-89.

Zhurbenko M. P. \& Zheludeva, E. V. 2015. Lichenicolous fungi from Russia, mainly from the Magadan Region. Folia Cryptogamica Estonica 52: 101-107. https://doi.org/10.12697/fce.2015.52.13

Zhurbenko, M. P., Chesnokov, S. V. \& Konoreva, L. A. 2016. Lichenicolous fungi from Kodar Range, Trans-Baikal Territory of Russia. Folia Cryptogamica Estonica 53: 9-22. https:/ / doi.org/10.12697/ fce.2016.53.02

Zhurbenko, M. P., Enkhtuya, O. \& Javkhlan, S. 2019. A first synopsis of lichenicolous fungi of Mongolia, with the description of five new species. Plant and Fungal Systematics 64(2): 345-366. https://doi. org/ $10.2478 /$ pfs-2019-0023 